\title{
Ro 90-7501 Is a Novel Radiosensitizer for Cervical Cancer Cells that Inhibits ATM Phosphorylation
}

\author{
KEISUKE TAMARI ${ }^{1}$, KEISUKE SANO $^{1,2}$, ZHIHAO LI $^{1}$, YUJI SEO ${ }^{1}$, KEISUKE OTANI $^{1}$, \\ SHOTARO TATEKAWA ${ }^{1}$, MASAYASU TORATANI ${ }^{1}$, YUJI TAKAOKA $^{1}$, YUTAKA TAKAHASHI ${ }^{1}$, \\ KAZUMASA MINAMI ${ }^{1}$, FUMIAKI ISOHASHI ${ }^{1}$, MASAHIKO KOIZUMI ${ }^{2}$ and KAZUHIKO OGAWA ${ }^{1}$ \\ Departments of ${ }^{1}$ Radiation Oncology and ${ }^{2}$ Medical Physics and Engineering, \\ Osaka University Graduate School of Medicine, Osaka, Japan
}

\begin{abstract}
Background/Aim: Ro 90-7501 has been reported as an inhibitor of the amyloid $\beta 42$ fibril assembly that is associated with Alzheimer's disease. The present study aimed to elucidate the radiosensitizing effects of Ro 90-7501 and focused on ATM signaling after irradiation. Materials and Methods: Clonogenic survival, apoptosis, and cell-cycle assays as well as western blotting were performed in HeLa cells treated with irradiation and Ro 90-7501. Tumor growth delay assay was also performed using BALB/c-nu mice. Results: The combination of irradiation with Ro 90-7501 showed significant radiosensitizing effects in clonogenic survival and tumor growth delay assays. Ro 90-7501 significantly increased apoptosis and impaired cell cycle after irradiation. Western blotting showed that Ro 90-7501 suppressed the phosphorylation of ATM and its downstream proteins, such as $H 2 A X, C h k 1$, and Chk2, after irradiation. Conclusion: Ro 90-7501 inhibits DNA damage response by inhibiting ATM and has significant radiosensitizing effects on cervical cancer cells.
\end{abstract}

Cervical cancer is one of the most common cancers among women worldwide, accounting for 569,847 new cases and 311,365 deaths annually (1). Radiotherapy has been commonly used in cervical cancer treatment for a long time, especially for locally advanced cancer. However, because treatment results are not satisfactory, development of radiosensitizers is one of the strategies to improve efficacy of radiotherapy. Although cisplatin is used as a radiosensitizer for locally advanced cervical cancer, patients often develop hematologic and gastrointestinal complications (2). Discovery

Correspondence to: Keisuke Tamari, Department of Radiation Oncology, Osaka University Graduate School of Medicine, 2-2 Yamadaoka, Suita, Osaka 565-0871, Japan. Tel: +81 668793482, e-mail: tamari@radonc.med.osaka-u.ac.jp

Key Words: Ro 90-7501, radiosensitizer, cervical cancer, ATM. for new radiosensitizers that are less toxic and more effective against cancer could improve treatment efficacy. In this study, we focused on the compound "Ro 90-7501", which is reported to be an inhibitor of amyloid $\beta 42$ fibril assembly involved in Alzheimer's disease (3).

Ataxia telangiectasia is caused by loss of ataxia telangiectasia mutated (ATM) function and is characterized by increased radiosensitivity (4). The ATM protein kinase is known as a tumor suppressor, which is frequently mutated in human cancers. ATM regulates cellular response to DNA double-strand breaks by phosphorylating various proteins such as Chk1, Chk2, and H2AX involved in DNA damage response and cell-cycle checkpoint activation after irradiation (5). Inhibition of DNA damage response can be effective in enhancing cytotoxicity after irradiation (6). In this context, the present study aimed to elucidate the effects of radiosensitization of Ro 90-7501 on DNA damage response through ATM signaling.

\section{Materials and Methods}

Cell culture. The human cervical cancer cell lines HeLa and ME180 were purchased from American Type Culture Collection (VA, USA) and were cultured in Dulbecco's modified eagle's medium supplemented with $10 \%$ fetal bovine serum (FBS), $100 \mathrm{U} / \mathrm{ml}$ penicillin, and $100 \mu \mathrm{g} / \mathrm{ml}$ streptomycin. Ro 90-7501 was purchased from Sigma (MO, USA) and dissolved in DMSO.

Irradiation. Cells were irradiated under ambient conditions using a ceasium-137 gamma-ray irradiator, Gammacell@ 40 Exactor (MDS Nordion, Ottawa, Canada) at the Osaka University Graduate School of Medicine with a delivering dose rate of approximately 0.85 $\mathrm{Gy} / \mathrm{min}$. The mice were irradiated using an orthovoltage X-ray irradiator (Rigaku Denki, Tokyo, Japan) under conditions of $180 \mathrm{kV}$ and $15 \mathrm{~mA}$ at the Institute of Experimental Animal Sciences Faculty of Medicine, Osaka University.

WST-1 assay. A total of 500 cells were plated per well in 96-well plates. Cells in each well were treated with several concentrations of Ro 90-7501. WST-1 solution $(10 \mu \mathrm{l})$ was added to each well 
$48 \mathrm{~h}$ later and the cells were then incubated for $1 \mathrm{~h}$. After incubation, absorbance was measured at $450 \mathrm{~nm}$ using a microplate reader (Bio-Rad, CA, USA).

Clonogenic survival assay. Cells were plated into $60 \mathrm{~mm}$-diameter dishes, treated with $10 \mu \mathrm{M}$ Ro 90-7501, and irradiated $4 \mathrm{~h}$ later. The medium was replaced with fresh medium $24 \mathrm{~h}$ after irradiation. The cells were fixed and stained with crystal violet, 10-14 days after irradiation. Colonies of more than 50 cells were counted and normalized according to the nonirradiated controls for each treatment.

Apoptosis assay. Apoptotic cells were analyzed using Annexin VFITC Apoptosis Detection Kit (Nacalai tesque, Kyoto, Japan). In brief, $24 \mathrm{~h}$ after 8 Gy irradiation, HeLa cells were washed in PBS and suspended in annexin V binding solution (1x). Annexin V-FITC solution and propidium iodide (PI) solution were then added to the cells. After incubation in the dark for $15 \mathrm{~min}$, cells were analyzed with BD FACS Canto II (BD Biosciences, NJ, USA).

Tumor growth delay assay. Eight-week-old female BALB/c nude mice were purchased from Charles River Laboratories Japan (Kanagawa, Japan). Mice were maintained under clean conventional conditions at Osaka University Graduate School of Medicine's Institute for Experimental Animal Sciences. All animal experiments were performed with the approval of Animal Experiments Committee of the Osaka University. A total of $1.5 \times 10^{7} \mathrm{HeLa}$ cells in $100 \mu \mathrm{l}$ PBS were inoculated into the legs of mice. Treatments were applied when tumors grew to $10 \mathrm{~mm}$. The mice were divided into four groups: control group (1\% DMSO/PBS i.p., 0 Gy), Ro 907501 group ( $5 \mu \mathrm{g} / \mathrm{g}$ of Ro $90-7501$ i.p., 0 Gy), irradiation group (1\% DMSO/PBS i.p., $10 \mathrm{~Gy})$, and combination group ( $5 \mu \mathrm{g} / \mathrm{g}$ of Ro $90-$ 7501 i.p., 10 Gy). Tumor diameters were measured using digital calipers, and tumor volume was calculated using the formula:

volume $=(\text { width })^{2} \times$ length $\times 0.5$

Western blotting. Western blotting was performed using standard protocols. After irradiation, cells were harvested using trypsin, washed twice with PBS, and lysed in a radioimmunoprecipitation assay lysis buffer (89900, Thermo Fisher Scientific; $25 \mathrm{mM}$ Tris $\bullet \mathrm{HCl}$ $\mathrm{pH} 7.6,150 \mathrm{mM} \mathrm{NaCl}, 1 \% \mathrm{NP}-40,1 \%$ sodium deoxycholate, $0.1 \%$ sodium dodecyl sulfate) containing protease inhibitor cocktail and phosphatase inhibitors. Equal amounts of protein $(30 \mu \mathrm{g})$ were loaded onto $10 \%$ SDS polyacrylamide gel. After electrophoresis, polyvinylidene difluoride membranes were blocked for $1 \mathrm{~h}$ at room temperature with $5 \%$ skim milk in tris-buffered saline with Tween 20 (TBST), followed by incubation for $16 \mathrm{~h}$ at $4^{\circ} \mathrm{C}$ with primary antibodies. The membranes were then incubated for $1 \mathrm{~h}$ at $23^{\circ} \mathrm{C}$ with 1:100,000 HRP conjugated secondary antibodies (111-035-144 for anti-rabbit, Jackson ImmunoResearch, PA, USA). After three additional washes with TBST for $10 \mathrm{~min}$, the signal was developed using the Amersham ECL Prime Western blotting detection reagent (RPN2232, GE Healthcare, Little Chalfont, UK) through incubation at $23^{\circ} \mathrm{C}$ for $5 \mathrm{~min}$. Finally, the signal was detected using ChemiDoc Touch Imaging System (Bio-Rad, CA, USA). The primary antibodies used were: 1:1000 anti-ATM (\#2873, CST), 1:1,000 anti-phosphoATM(Ser1981) (\#5883, CST), 1:500 anti-phospho-ATR(Ser428) (\#2853, CST), 1:1,000 anti-phospho-Histon H2A.X(Ser139) (\#9718, CST), 1:1,000 anti-phospho-Chk1(Ser345) (\#2348, CST), 1:1,000
anti-phospho-Chk2(Thr68) (\#2197, CST), and 1:1,000 beta-actin (\#4870, CST).

Cell cycle analysis. Cells were harvested using trypsin, washed with cold PBS, and fixed with $70 \%$ ethanol at $-20^{\circ} \mathrm{C}$ overnight. Subsequently, the cells were resuspended in PI and RNase in PBS at room temperature in the dark for $30 \mathrm{~min}$. The stained cells were analyzed using FACS canto II (BD Biosciences).

Statistical analysis. The results were expressed as mean \pm standard deviations. Statistical significance was analyzed using the Student's $t$-test. A $p$-value of $<0.05$ was considered statistically significant.

\section{Results}

Ro 90-7501 enhanced radiosensitivity and apoptosis in cervical cancer cell lines. To examine the radiosensitizing effects of Ro 90-7501 on cancer cells, we performed clonogenic survival assays using HeLa and ME-180 cells. Ro 90-7501 significantly enhanced radiosensitivity compared with control HeLa and ME-180 cells (Figure 1A). The effect of Ro 90-7501 on cell toxicity is presented in Figure 1B. An apoptosis assay showed that 8 Gy irradiation with Ro 907501 significantly increased the number of apoptotic cells compared with 8 Gy irradiation alone. Interestingly, Ro 907501 alone did not significantly increase the number of apoptotic cells compared with the control (Figure 2A and B).

Delay of tumor growth using irradiation and Ro 90-7501. To examine the radiosensitizing effects of Ro 90-7501 in vivo, HeLa cells were inoculated in the legs of BALB-c nude mice and tumor volumes were measured in the control, Ro 907501 alone, irradiation alone, and combination groups. To account for differences in the volume of the inoculated cells, each tumor volume was normalized to that at day 0. As shown in Figure 3, tumor growth was significantly delayed in the combination group compared with the other groups at 21 days after treatment. Tumor volume was also significantly decreased in the irradiation group compared with the control and Ro 90-7501 groups at 21 days after treatment. In addition, there was no difference in tumor volume between the Ro 90-7501 and control groups at 21 days after treatment. Taken together, our results suggest that Ro 907501 had a radiosensitizing effect in vivo as well as in vitro.

Ro 90-7501 inhibits phosphorylation of ATM, and its downstream proteins. To investigate the mechanism by which Ro 90-7501 enhances radiosensitivity, we examined the levels of proteins involved in DNA damage response using western blotting (Figure 4). The levels of phosphorylated $\mathrm{H} 2 \mathrm{AX}(\mathrm{pH} 2 \mathrm{AX})$ gradually increased at $2 \mathrm{~h}$ after irradiation but then they decreased. Interestingly, $\mathrm{pH} 2 \mathrm{AX}$ was suppressed in the combination group compared with the radiation group. We hypothesized that ATM was inhibited by Ro 90-7501 because ATM is known to 

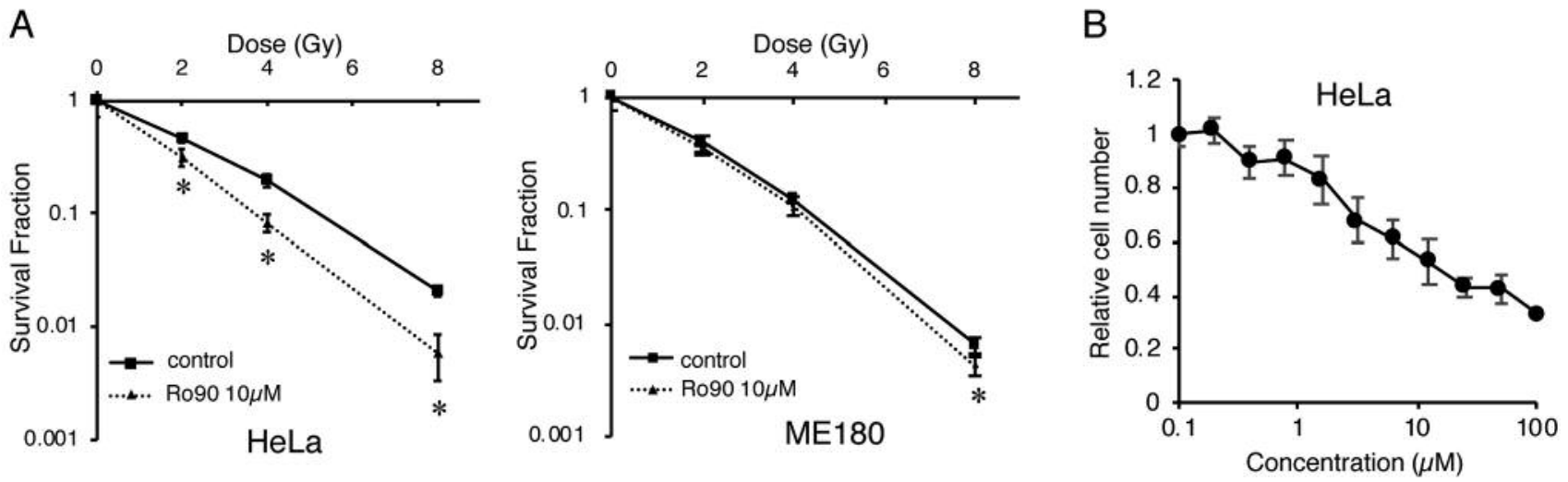

Figure 1. Survival fractions in HeLa and ME-180 cells irradiated with or without Ro 90-7501. Evaluation of radiosensitivity with or without Ro 90-7501 in HeLa and ME-180 cells (A). Cell toxicity assay was performed in cells treated with Ro 90-7501(B). Results were normalized by the survival of nonirradiated cells. Each graph presents the mean \pm standard deviation. *: $p<0.05$.

A

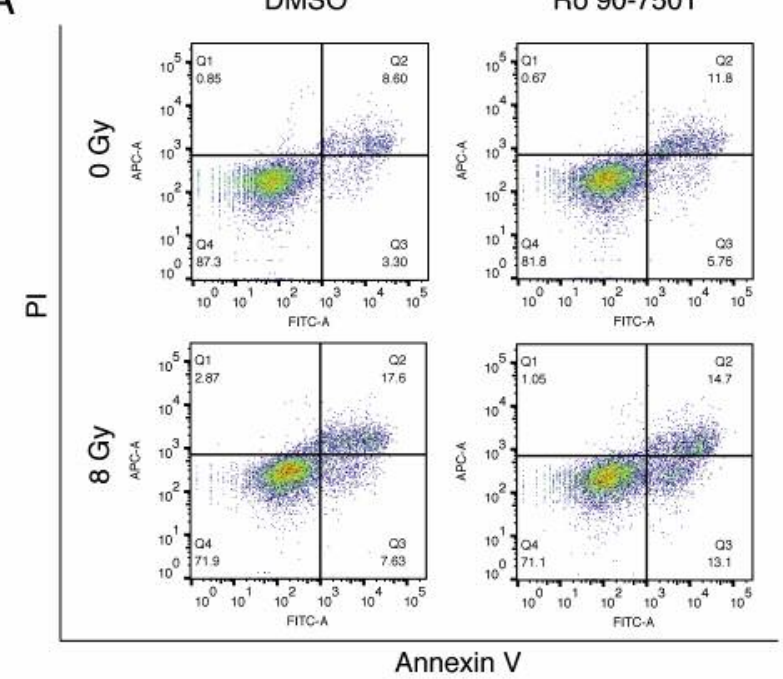

B

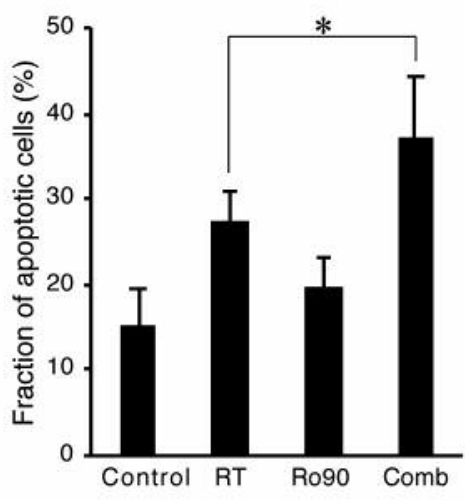

Figure 2. Induction of apoptosis by Ro 90-7501 and radiation in HeLa cells. (A) Cells were treated with Ro 90-7501 for 2 h prior to treatment with 8 Gy irradiation. Apoptosis was measured using propidium iodide (PI)/annexin-V double staining of Hela cells after 24 h irradiation. (B) Data are presented as mean \pm standard deviation. *: $p<0.05$.

phosphorylate $\mathrm{H} 2 \mathrm{AX}$ in response to DNA double-strand breaks (7). The levels of phosphorylated ATM (pATM) rapidly increased in both irradiation and combination groups after treatment and were markedly decreased at $2 \mathrm{~h}$ after irradiation in the combination group. In addition, the levels of phosphorylated Chk1 (pChk1) and Chk2 (pChk2), which are known downstream targets of ATM, also increased rapidly after irradiation and, interestingly, were suppressed in the combination group. These results suggest that Ro 907501 suppresses the phosphorylation of ATM and its downstream targets.
Ro 90-7501-induced cell cycle arrest after irradiation. Chk1 and Chk2 play a key role in cell cycle progression (8). Given that pChk1 and pChk2 were suppressed in the combination group, cell cycle should have been disturbed in the combination treatment. To analyze the effects of Ro 90-7501 on cell cycle, a cell cycle analysis was performed at $24 \mathrm{~h}$ after 8 Gy irradiation using HeLa cells. In the combination group, cells were exposed to $10 \mu \mathrm{M}$ Ro 90-7501 for $2 \mathrm{~h}$ before irradiation. Compared with the control, Ro 90-7501 decreased the population of cells in the $G_{1}$ and $S$ phase of cell cycle and increased that in the $\mathrm{G}_{2} / \mathrm{M}$. Compared with the 


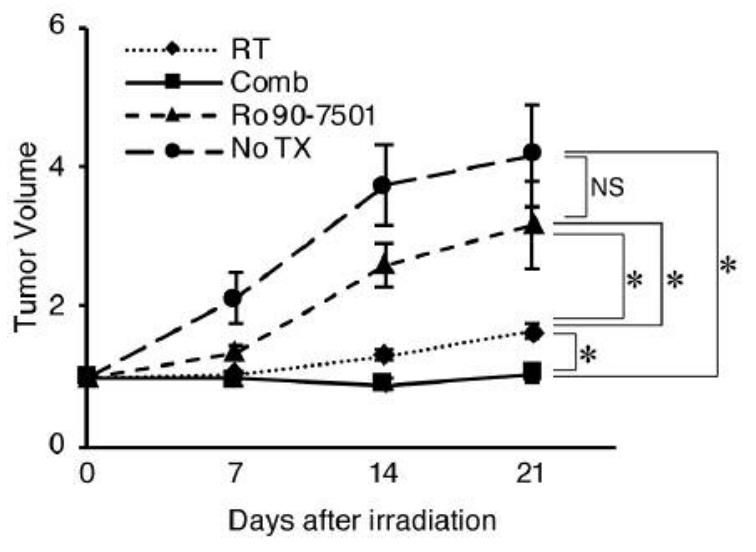

Figure 3. The effects of Ro 90-7501 on in vivo tumor growth in control $(N=6)$, Ro 90-7501 $(N=8), 8 \mathrm{~Gy}(N=16)$, and Ro 90-7501 with $8 \mathrm{~Gy}$ $(C o m b, N=16)$ mice. Each volume was normalized by the volume of the tumor at day 0 . Data are presented as mean \pm standard deviation. $* p<0.05$.

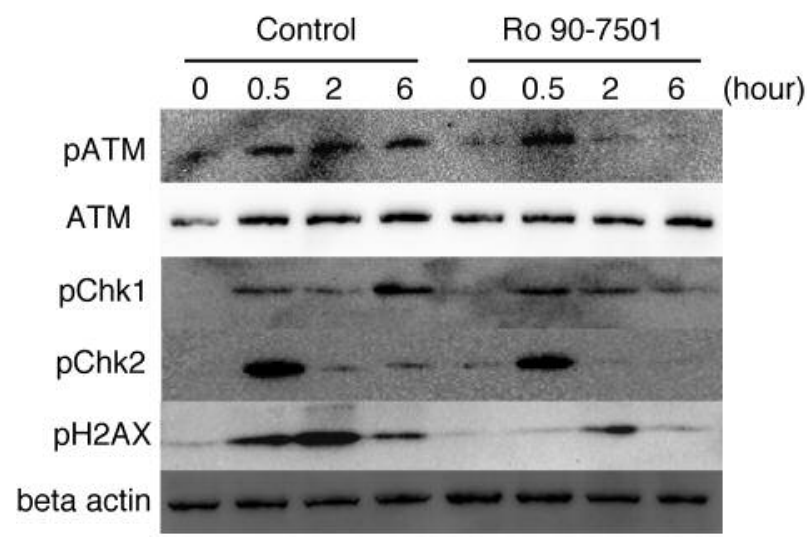

Figure 4. Ro 90-7501 inhibits the phosphorylation of proteins responsible for DNA damage repair. Western blotting using antibodies of $p H 2 A X, p-A T M, A T M, p-A T R, p$-Chk1, and p-Chk2 was performed at 0, 0.5, 2, and 6 h after 8 Gy irradiation with or without Ro 90-7501.
A

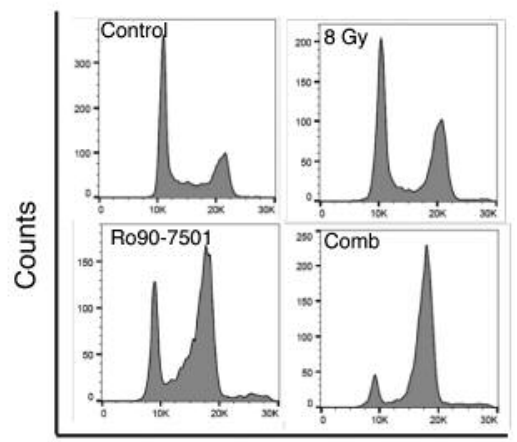

DNA content

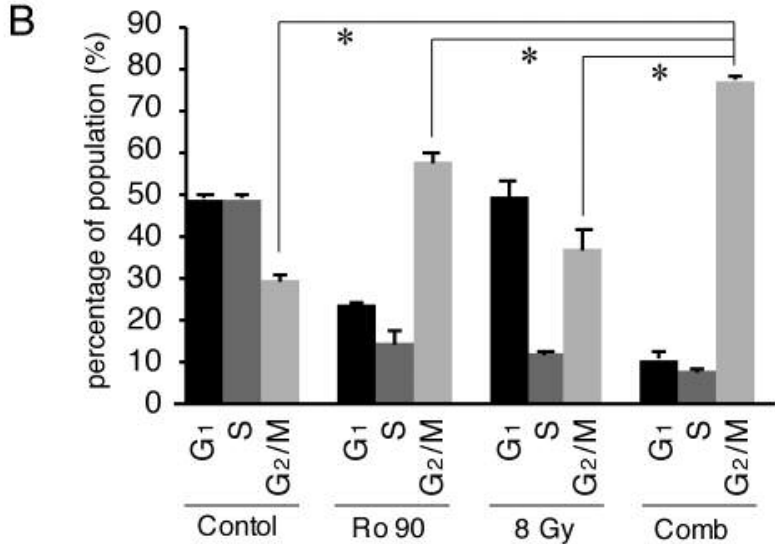

Figure 5. The effects of Ro 90-7501 and radiation on the cell cycle of HeLa cells. (A, B) HeLa cells were treated with 10 uM of Ro $90-7501$ for $2 \mathrm{~h}$ before $8 \mathrm{~Gy}$ irradiation. After $24 \mathrm{~h}$, cells were harvested and analyzed. The data are presented as mean \pm standard deviation. ${ }^{*} p<0.05$.

control, irradiation increased the population of cells in the $\mathrm{G}_{1}$ phase and decreased those in the $S$ and $G_{2} / M$ phases. Interestingly, the combination treatment significantly increased the population of cells in the $\mathrm{G}_{2} / \mathrm{M}$ phase and decreased that in the $G_{1}$ phase compared with the control, irradiation, and Ro 90-7501 groups (Figure 5A and B). These results suggest that inhibition of phosphorylation of Chk1 and pChk2 has a significant effect on $\mathrm{G}_{2} / \mathrm{M}$ arrest.

\section{Discussion}

In this study, we demonstrated that Ro 90-7501 enhances the radiosensitivity of cervical cancer cells both in vitro and in vivo. Treatment resistance in cancer is known to be caused by tumor heterogeneity. Cancer stem cells and hypoxic cells that exist among cancer cells play a role in radioresistance $(9,10)$. Radiosensitizers, which enhance the cytotoxic effect of radiotherapy, have been studied for a long time, especially in terms of their effect on hypoxic cancer cells. Nimorazole, a well-known radiosensitizer targeting hypoxic tumors, has been used in radiotherapy for head and neck cancer treatment (11). Recently, ATM inhibitors have been used as radiosensitizers $(12,13)$ and their efficacy in eradicating radioresistant glioma stem cells has been reported (14). In the present study, the experiments in mice demonstrated no apparent toxicities after administration of Ro 90-7501 alone or in combination with irradiation. ATM inhibitors may not increase the toxicity of irradiation to normal tissue. Herzog 


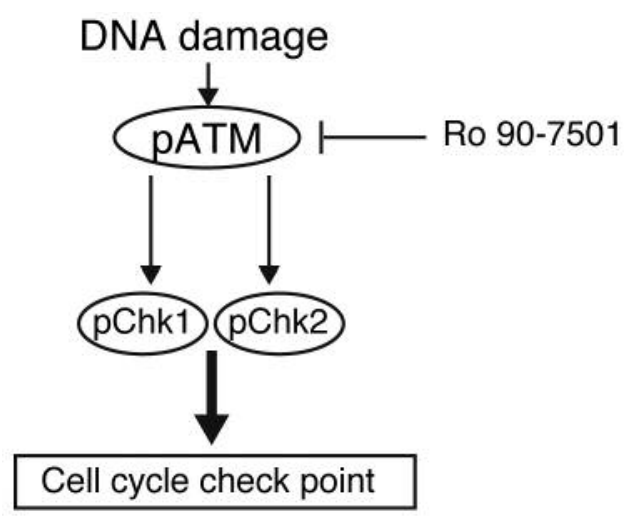

Figure 6. Radiosensitization mechanism of Ro 90-7501. Ro 90-7501 induced cell-cycle arrest after irradiation by suppressing ATM and its downstream protein phosphorylation.

KH et al. have demonstrated that $\mathrm{Atm}^{-/-}$mice developed resistance to apoptosis in the central nervous system after ionizing radiation (15). Moding E et al. have demonstrated that heart endothelial cells of $\mathrm{Atm}^{-/-}$mice did not develop radiosensitivity (16).

Recently, Ro 90-7501 has been reported to inhibit the action of protein phosphatase 5 (PP5), which is a serine/threonine phosphatase that belongs to the PPP family phosphatases (17). PP5 is known to play a role in ATM activation after irradiation (18). These findings suggest that Ro 90-7501 inhibits ATM through PP5 inhibition. Drug repositioning is a field of drug discovery that identifies new therapeutic indications for existing drugs (19). Because Ro 90-7501 has been reported as an inhibitor of amyloid $\beta 42$ fibril assembly (3), we propose Ro 90-7501 to be repositioned as an ATM inhibitor. In conclusion, the present study demonstrated that Ro 90-7501 has significant radiosensitizing effects on cervical cancer cells. It inhibits the phosphorylation of ATM and its downstream proteins, suppress cell cycle progression, and increases apoptosis after irradiation (Figure 6).

\section{Conflicts of Interest}

The Authors have no conflicts of interest to declare regarding this study.

\section{Authors' Contributions}

Keisuke Tamari: Data acquisition, Original Draft preparation; Keisuke Sano and Zhihao Li: Data acquisition; Yuji Seo, Kazumasa Minami, Yutaka Takahashi, Shotaro Tatekawa, Masayasu Toratani, Yuji Takaoka, and Keisuke Otani: Data analysis and interpretation; Fumiaki Isohashi: Study design; Masahiko Koizumi and Kazuhiko Ogawa: Review and Editing, Supervision.

\section{Acknowledgements}

The Authors would sincerely like to thank the entire laboratory staff for the fruitful discussions and Yoon-Jeong Kim and Kazuto Nunomura for their technical assistance.

\section{References}

1 Bray F, Ferlay J, Soerjomataram I, Siegel RL, Torre LA and Jemal A: Global cancer statistics 2018: GLOBOCAN estimates of incidence and mortality worldwide for 36 cancers in 185 countries. CA Cancer J Clin 68(6): 394-424, 2018. PMID: 30207593. DOI: $10.3322 /$ caac. 21492

2 Jakubowicz J, Blecharz P, Skotnicki P, Reinfuss M, Walasek T and Luczynska E: Toxicity of concurrent chemoradiotherapy for locally advanced cervical cancer. Eur J Gynaecol Oncol 35: 393399, 2014. PMID: 25118480.

3 Bohrmann B, Adrian M, Dubochet J, Kuner P, Müller F, Huber W, Nordstedt C and Döbeli H: Self-assembly of beta-amyloid 42 is retarded by small molecular ligands at the stage of structural intermediates. J Struct Biol 130: 232-246, 2000. PMID: 10940228. DOI: $10.1006 /$ jsbi.2000.4241

4 Painter RB and Young BR: Radiosensitivity in ataxia-telangiectasia: a new explanation. Proc Natl Acad Sci USA 77: 73157317, 1980. PMID: 6938978. DOI: 10.1073/pnas. 77.12.7315

5 Shiloh Y and Ziv Y: The ATM protein kinase: regulating the cellular response to genotoxic stress, and more. Nat Rev Mol Cell Biol 14: 197-210, 2013. PMID: 23486281. DOI: 10.1038/ nrm3546

6 Goldstein $M$ and Kastan MB: The DNA damage response: Implications for tumor responses to radiation and chemotherapy. Annu Rev Med 66: 129-143, 2015. PMID: 25423595. DOI: 10.1146/annurev-med-081313-121208

7 Burma S, Chen BP, Murphy M, Kurimasa A and Chen DJ: ATM phosphorylates histone H2AX in response to DNA double-strand breaks. J Biol Chem 276: 42462-42467, 2001. PMID: 11571274. DOI: $10.1074 /$ jbc.C100466200

8 Bartek $\mathrm{J}$ and Lukas $\mathrm{J}$ : Chk1 and Chk2 kinases in checkpoint control and cancer. Cancer Cell 3: 421-429, 2003. PMID: 12781359.

9 Bachtiary B, Schindl M, Pötter R, Dreier B, Knocke TH, Hainfellner JA, Horvat R and Birner P: Overexpression of hypoxia-inducible factor 1 alpha indicates diminished response to radiotherapy and unfavorable prognosis in patients receiving radical radiotherapy for cervical cancer. Clin Cancer Res 9: 2234-2240, 2003. PMID: 12796391.

10 Hayashi K, Tamari K, Ishii H, Konno M, Nishida N, Kawamoto K, Koseki J, Fukusumi T, Kano Y, Nishikawa S, Miyo M, Noguchi K, Ogawa H, Hamabe A, Seo Y, Doki Y, Mori M and Ogawa K: Visualization and characterization of cancer stem-like cells in cervical cancer. Int J Oncol 45: 2468-2474, 2014. PMID: 25269542. DOI: 10.3892/ijo.2014.2670

11 Overgaard J, Hansen HS, Overgaard M, Bastholt L, Berthelsen A, Specht L, Lindeløv B and Jørgensen K: A randomized double-blind phase III study of nimorazole as a hypoxic radiosensitizer of primary radiotherapy in supraglottic larynx and pharynx carcinoma. Results of the Danish Head and Neck Cancer Study (DAHANCA) Protocol 5-85. Radiother Oncol 46: 135-146, 1998. PMID: 9510041. 
12 White JS, Choi S and Bakkenist CJ: Transient ATM kinase inhibition disrupts DNA damage-induced sister chromatid exchange. Sci Signal 3: 1-12, 2010. PMID: 20516478. DOI: 10.1126/scisignal.2000758

13 Dohmen AJC, Qiao X, Duursma A, Wijdeven RH, Lieftink C, Hageman F, Morris B, Halonen P, Vens C, van den Brekel MWM, Ovaa H, Neefjes $J$ and Zuur CL: Identification of a novel ATM inhibitor with cancer cell specific radiosensitization activity. Oncotarget 8: 73925-73937, 2017. PMID: 29088757. DOI: $10.18632 /$ oncotarget.18034

14 Ahmed SU, Carruthers R, Gilmour L, Yildirim S, Watts C and Chalmers AJ: Selective inhibition of parallel DNA damage response pathways optimizes radiosensitization of glioblastoma stem-like cells. Cancer Res 75: 4416-4428, 2015. PMID: 26282173. DOI: $10.1158 / 0008-5472$.CAN-14-3790

15 Herzog KH, Chong MJ, Kapsetaki M, Morgan JI and McKinnon PJ: Requirement for Atm in ionizing radiation-induced cell death in the developing central nervous system. Science 280: 10891091, 1998. PMID: 9582124. DOI: 10.1126/science.280. 5366.1089

16 Moding EJ, Lee CL, Castle KD, Oh P, Mao L, Zha S, Min HD, Ma Y, Das S and Kirsch DG: ATM deletion with dual recombinase technology preferentially radiosensitizes tumor endothelium. J Clin Invest 124: 3325-3338, 2014. PMID: 25036710. DOI: $10.1172 /$ JCI73932
17 Hong TJ, Park K, Choi EW and Hahn JS: Ro 90-7501 inhibits PP5 through a novel, TPR-dependent mechanism. Biochem Biophys Res Commun 482: 215-220, 2017. PMID: 27840051. DOI: $10.1016 /$ j.bbrc.2016.11.043

18 Ali A, Zhang J, Bao S, Liu I, Otterness D, Dean NM, Abraham RT and Wang XF: Requirement of protein phosphatase 5 in DNA-damage-induced ATM activation. Genes Dev 18: 249-254, 2004. PMID: 14871926. DOI: 10.1101/gad.1176004

19 Ashburn TT and Thor KB: Drug repositioning: Identifying and developing new uses for existing drugs. Nat Rev Drug Discov 3: 673-683, 2004. PMID: 15286734 . DOI: $10.1038 / \mathrm{nrd} 1468$

Received July 11, 2019

Revised August 9, 2019 Accepted August 14, 2019 